\title{
XLVII. On the relative ionization potentials of gases as observed in thermionic valves
}

\section{G. Stead M.A. \& B.S. Gossling M.A.}

To cite this article: G. Stead M.A. \& B.S. Gossling M.A. (1920) XLVII. On the relative ionization potentials of gases as observed in thermionic valves, Philosophical Magazine Series 6, 40:238, 413-425, DOI: $10.1080 / 14786441008636142$

To link to this article: http://dx.doi.org/10.1080/14786441008636142

曲 Published online: 08 Apr 2009.

Submit your article to this journal $₫$

Џ Article views: 3

Q View related articles $\square$

Citing articles: 1 View citing articles 5 
It has been urged against the idea of a value of force not varying inversely as the square of the distance that the scattering of the a particles indicates a force of this type. The scattering of the $\alpha$ particles, however, practically all takes place at distances from the centre very small compared with the distance of the electrons which scatter the light or affect the emission of visible light; and it is quite possible that the law should be the inverse square both at distances which are very large compared with $c$ and again at distances which are very small compared with it, and yet be quite of a different character when $r$ is comparable with $c$-in fact, the law expressed by the value of $\phi$ given above is at both very great and very small distances that of the inverse square.

XLVII. On the Relative Ionization Potentials of Gases as observed in Thermionic Valves. By G. STEAD, M.A., and B. S. Gossling, M.A.*

PrelimIN.lRy Note. - The observations described in this paper were made during the summer of 1917 , in connexion with the improvement of thermionic valves of a type in which the action was in considerable measure dependent on ionization. They are now published with the permission of the Admiralty.

(1) Method employed.-Some of the earlier methods $\dagger$ of determining the ionization potentials of gases have been the subject of criticism as indicating, not the appearance of positive ions, but some other effect such as photo-electric emission of electrons from the electrodes whose intended function was to collect the positive ions when formed. In the experiments here described the method used was similar to that employed by Bazzoni $\ddagger$, and consists in observing the effects of the presence of gas on the form of curves representing the variation of the current leaving an incandescent cathode with change in the potential applied to the collecting electrodes. When no gas is present, the chief factor determining the value of the space-current is the modification of the collecting field due to the applied potential by the addition

* Communicated by Professor Sir J. J. Thomson.

$+E$. g. Frauck and Hertz, Deutsch. Phys. Ges, vol. xv. (1913); Pawlow, Proc. Roy. Soc. vol. xc. p. 398 (1914).

$\ddagger$ Phil. Mag. vol. sxxii. p. 566 (1916). 


\section{Messrs. Stead and Gossling on Relative Ionizalion}

of the field due to the electrons at the time in transit across the space. (Space-charge effect.) If the electrons are projected with negligible velocity from an equipotential cathode, it can be shown * that the current will vary as the $3 / 2$ power of the applied voltage up to the point when the current reaches a saturation value dependent on the temperature of the cathode, as shown in fig. 1. The current-voltage characteristics of tubes in which the voltage is great enough for the corrections for initial velocity and for variation in the

Fig. 1.

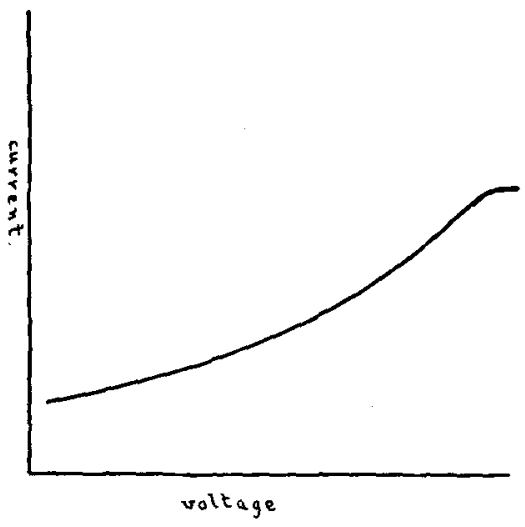

potential of the starting point on the cathode surface to be negligible, afford ample and accurate confirmation of this theory, which has further been applied with uniform success in the design of more complieated instruments, such as high-voltage thermionic valves.

When, however, the applied voltage is limited to low values, as in the case of the investigation of ionization effects, the effects of initial velocity and variation along the cathode are no longer to be neglected. In these experiments the same cathode heated to approximately the same temperature was used throughout. 'The results therefore indicate values of the ionization potential relative to any one of the gases observed taken as standard. It is, however, necessary to assume that replacement of one gas by another does not give rise to serious changes in the contact potential between the emitting and collecting electrodes.

The tube used was a three-electrode valve of the wellknown form, the cold electrodes being connected together for the purposes of the experiments. Next the tungsten

* Physical Review, vol. ii. p. 455 (1913). 
filament there was a spiral molybdenum wire of diameter $0.4 \mathrm{~mm}$. coiled into a helix of diameter $4.5 \mathrm{~mm}$., having four or five convolutions per cm. length. Ontside this was a nickel cylinder $10 \mathrm{~mm}$. in diameter. In such a tube if the vacuum is high enough for the effects of ionization to be inappreciable, it is necessary to apply a potential of some 60 volts to the combined collecting electrodes in order to reach the saturation value of the space-current when the cathode is, as in these experiments, hot enough to give a saturation current of 30 milliamperes. The lower parts of the current-voltage curve, well away from this saturation value, are observed to have a form sensibly independent of the cathode temperature, but showing a slight bodily movement, not accompanied by change of form, in the negative direction parallel to the potential axis when the temperature is increased.

This movement is ascribable in some measure to increase in the average velocity of projection of the electrons constituting the current, but in large part also to increase of the length of the active part of the filament owing to a closer approach to the negative end of the filament by the point where the filament surface, in spite of the cooling effect of the end supports, first becomes hot enough to emit.

If, however, gas is present in considerable quantity, a point can be found, by giving to the applied potential a

Fig. 2

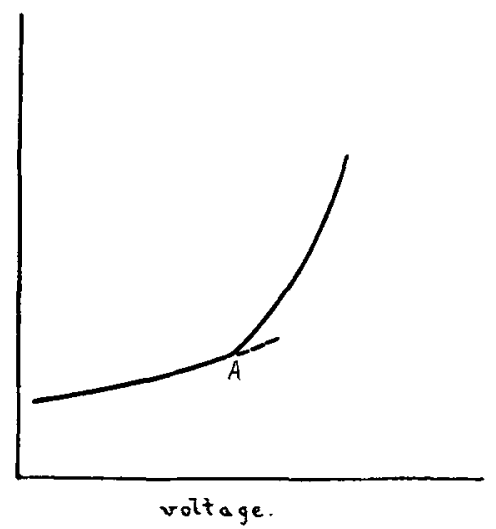

suitable value dependent on the gas used, where there is a marked departure from the form of the high-vacuum characteristic, an upward turn of the curve making its appearance, as shown at $\mathrm{A}$ in fig. 2. The high-vacuum 


\section{Messrs. Stead and Gossling on Relative Ionization}

characteristic is reproduced in the lower parts of the curve; but the subsequent upward turn indicates, of course, that the higher applied potentials are competent to produce a larger eurrent than in the high-vacuum case. In view of Langmuir's theory of the effect of the space-charye it seems difficult to account for this larger current except by the assumption that positive ions have appeared in the neighbourhood of the cathode. Positive ions would be expected to produce just such an effect as is observed, for they would tend to neutralize the opposing field due to the electrons in transit, and so permit the passage of a larger electron current for any given value of the applied voltage. As Professor Sir J. J. Thomson * has poiuted out in a slightly different connexion, a very small proportion of positive ions produces a marked effect on the tield by reason of their low velocity and consequent large contribution to the space-charge in their neighbourhood.

(2) Discussion of Method.-As mentioned above, some of the methods which have been used in attempts to measure ionization potentials are open to the serious objection that they fail to distinguish between the picking up of positive ions by the collecting electrodes and the photo-electric emission of negative electricity from these electrodes. The method described in the present paper is free from this objection, as the collecting electrodes are always at a positive potential with respect to the filament, and their function is to collect electrons, so that photo-electric emission of electrons would diminish rather than increase the current. If photoelectric emission from the filament itself is supposed to occur, this will merely slightly increase the saturation current from the filament, and will have no influence whatever on the current through the tube.

There remains the possibility that the sudden increase in the slope of the current-voltage curve is due to the production of positive ions, not by the thermions from the filament, but by more rapidly moving photo-electrons emitted by either the filament or the collecting electrodes under the influence of ultra-violet radiations from the gas molecules. Such a view, however, appears to be inconsistent with the quantum relation $\mathrm{V} e=h \nu$, for this relation not only determines the frequency of the ultra-violet light necessary to cause an emission

" Roy. Inst. Lecture, " Engineering," 103, p. 563 (1917). Proc. R. Inst. xxii. p. 175 (1917). 
of electrous having energy equal to $V e$, but also determines the energy which the bombarding electrons must have in order to stimulate the gas molecules to radiate ultra-violet light of frequency $\nu$. In other words, if the quantum relation holds, the photo-electrons could never possess more energy than the thermally emitted electrons which are responsible for the radiation of the ultra-violet light, so that photo-electric emission could not cause positive ions to appear until the velocity of the thermions was sufficient to cause ionization of the gas.

Finally it is to be noticed that the value of the current through the tube at the critical point is of the order of 5 milliamperes, and this in itself appears to render any photo-electric explanation very improbable, unless the marked increase in currents of this magnitude is taken as representing a photo-electric emission far larger than any hitherto describer.

(3) Experimental Results.-The method here deseribed for the determination of ionization potentials has been applied to six different gases, with the following results :-

\begin{tabular}{|c|c|}
\hline Mercury vapour ............ & $10 \cdot 8$ \\
\hline Argon & $12 \cdot 5$ \\
\hline Hydrogen & 15 \\
\hline Carbon monoxide ... & 15 \\
\hline Nitrogen & $17 \cdot 2$ \\
\hline Helium & $20 \cdot 8$ \\
\hline
\end{tabular}

The numbers given in the above table represent the difference of potential between the anode and the negative end of the filament, and have not been corrected in any way. A short discussion of the necessary corrections is given in Section (4) below. Specimen curves showing the relation between current and voltage for each of the six gases are shown in figs. 3 and 4.

A certain amount of advantage is obtained by plotting the logarithm of the current against the logarithm of the applied voltage, as the initial part of the curve, before ionization sets $i n$ is determined by the $3 / 2$ power of the voltage, so that it becomes a straight line when plotted logarithmically. The line ceases to be straight when positive ions begin to appear. The curves of fig. 4 are re-pletted logarithmically in fig, 5 . 
418 Messrs. Stead and Gos -ling on Relative Ionization

Fig. 3.

Current-voltage curves for valves containing argon, hydrogen and helium.

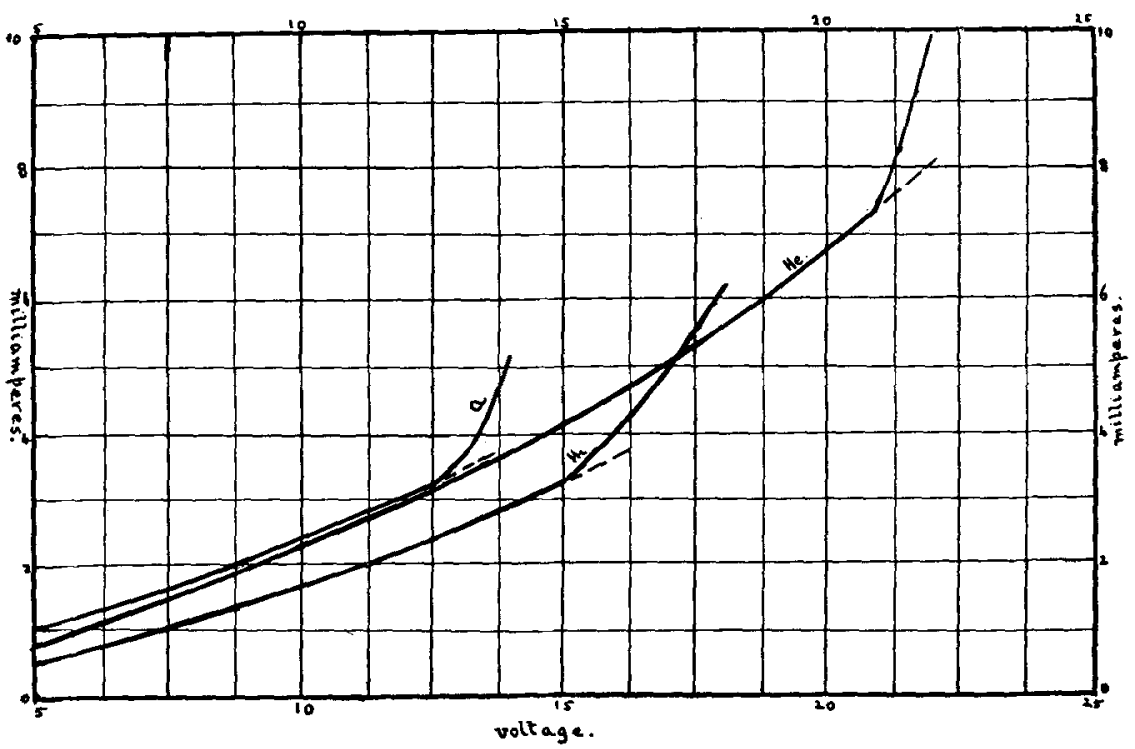

Fig. 4.

Curnent-voltoge curves for valves containing mercury vapour, carbon monoxide, and nutragen.

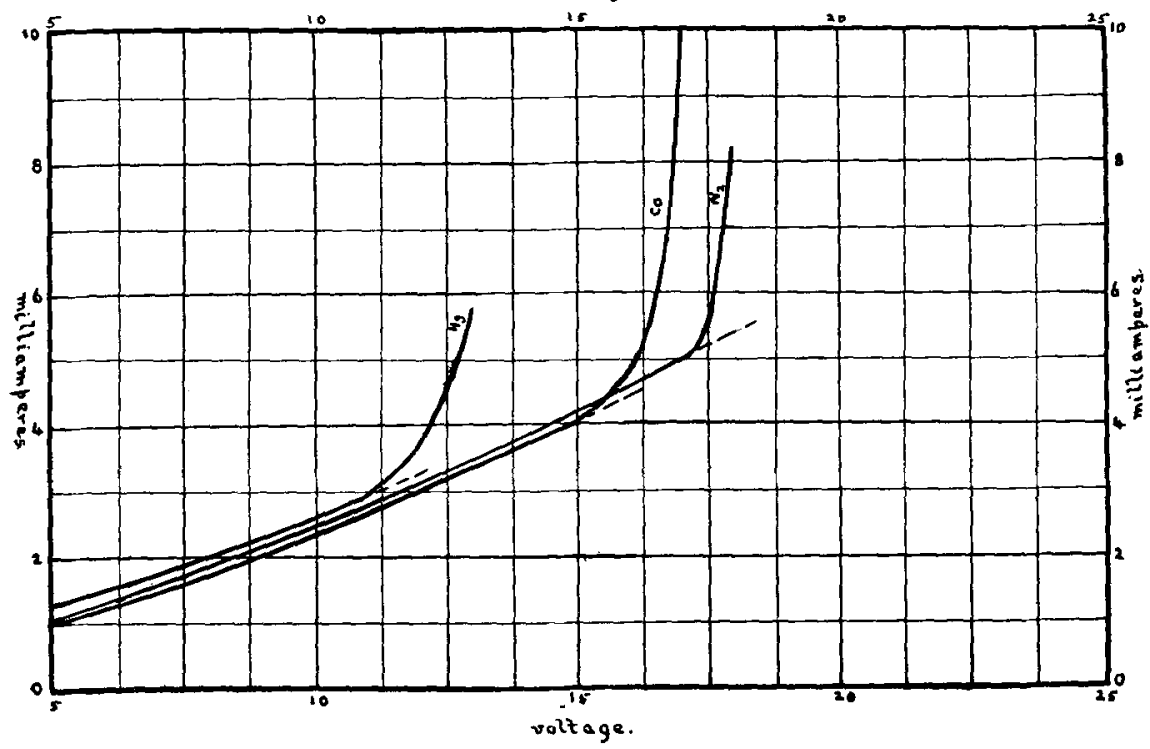


Potentials of Gases observed in Thermionic Valves. 419

Fig. 5.

Logarithinic current-voltage curves for mercury, sartion monoxide and

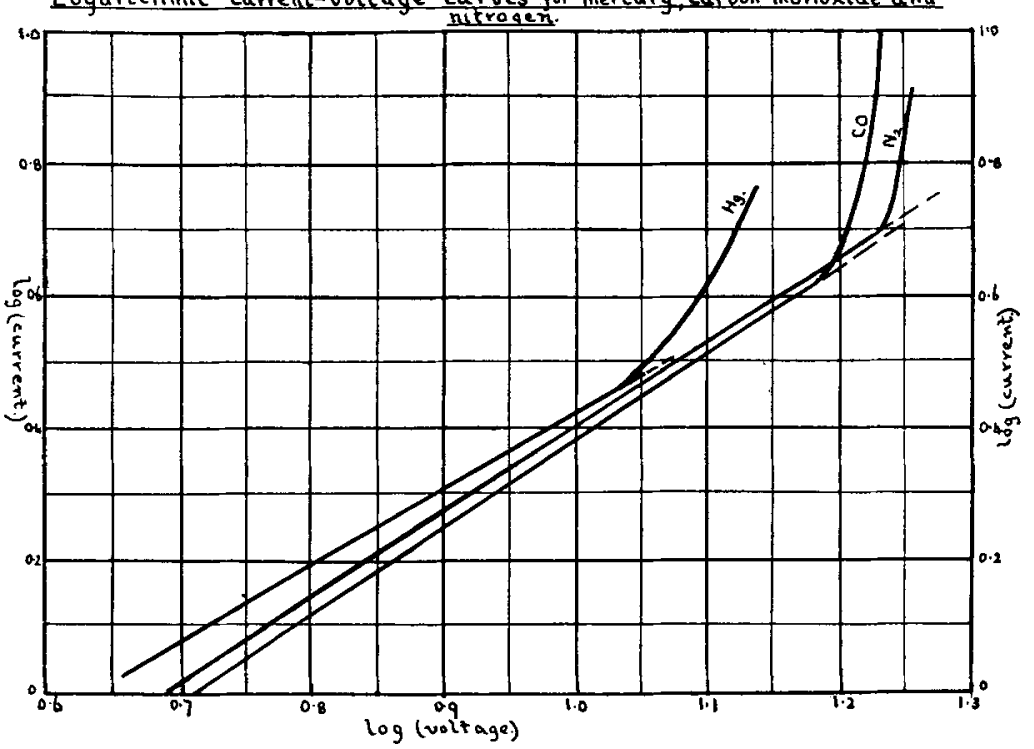

Fig. 6.

Current-voltage curves for filament in nitrogen at various pressures.

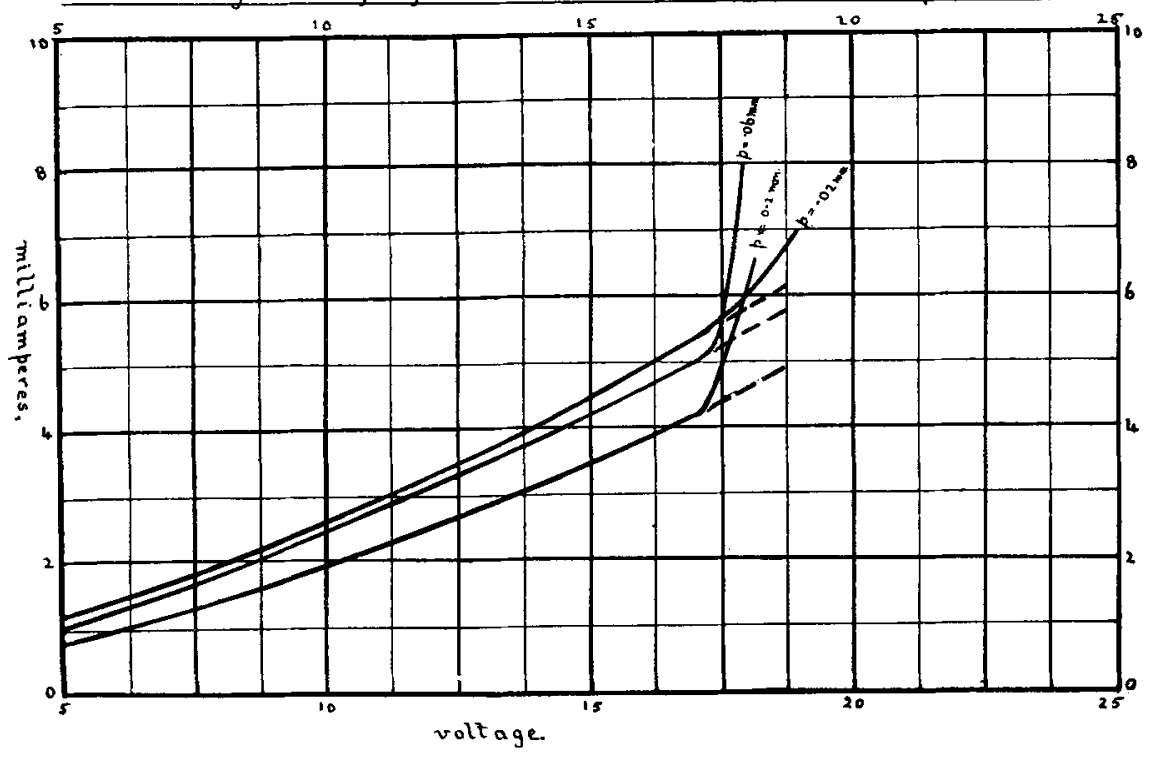




\section{Messrs. Stead and Gossling on Relative Ionization}

Experiments have been made with most of the six gases over a considerable range of pressure, and it has been shown that the critical point in the curvo, for any given gas, occurs at a voltage which is sensibly independent of the pressure. The change of slope at the critical point is, of course, dependent on the gas pressure, and becomes less marked as the pressure is diminished, but otherwise there is no alteration. This is brought ont clearly in fig. 6.

In all the experiments the valve used was highly exhausted by means of a Gaede rotary pump, followed by charcoal cooled by liquid air. During the evacuation the valve was baked at a temperature of about $400^{\circ}$ (Y. for at least an hour, and the cold electrodes were subjected to sufficient electron bombardment to raise them to a red heat, in order to remove occluded gas. The gas to be experimented upon was then introduced in as pure a state as possible. The argon used was prepared from liquid air residues by sparking with oxygen over caustic potash, the excess of oxygen being finilly removed by means of phosphorus. The argon when examined spectroscopically appeared to be free from nitrogen. Hydrogen was prepared by the electrolysis of a solution of barium hydroxide in water. Nitrogen from air was tried, but for most of the experiments it was prepared by mixing strong solutions of ammonium chloride and sodium nitrite and warming the mixture. Carbon monoxide was obtained by the action of strong sulphuric acid on formic acid, and also by heating nickel carbonyl. The helium used was obtained from Thomas Tyrer \& Co., Ltd., and was purified by being left in contact with charcoal cooled by liquid air for a considerable time. In all cases mercury vapour was excluded by placing betwen the valve and the pump a U-tube immersed in liquid air. When mercury vaponr itself was being studied this liquid air was removed after the valve had been thoroughly evacuated.

It may be remarked that, from the point of view of experiments on valves, and other apparatus in which a hot cathode is used, a knowledge of ionization potentials is often of great, service, as the presence of a given gas in a sealed valve may frequently be detected by measuring the ionization potential in this way. Moreover, the test is quite sensitive. Thus the presence of mercury vapour is readily shown, even when it cannot be detected spectroscopically. Of course the conditions in a valve are not good for spectroscopic observation, as the discharge obtained through the bulb by means of an induction-coil is not very bright, but, nevertheless, this gives some idea of the sensitiveness of the ionization test. Again, 
many valves which nominally contain helium, or nitrogen, give ionization voltages of about 15 after they have been in use for a short time. This indicates the presence of electrode gas, consisting probably of a mixture of carbon compounds and hydrogen, and shows that the bombardment of the anode and grid during the evacuation has not been carried far enough.

(4) Corrections.-The values of the ionization potentials obtained by the method here described require some correction, owing to the fact that the filament of a valve is not all at the same potential, nor at the same temperature, whilst correction is also necessary for the initial velocities of the emitted electrons, and for any contact potential difference which may exist between the filament and the collecting electrodes. As already remarked, the potential differences recorded are between the anode and the negative end of the filament, $i . e$. , they are the maximum potential difference in the valve. As a matter of fact the number of electrons which fall through this maximum difference of potential is entirely negligible, owing to the cooling of the ends of the filament by the leads. The magnitude of the cooling effect due to the leads has been fully discussed by one of the writers in a previous paper*; and it appear's that if a point is taken on the negarive limb of the filament such that the electron emission from it (per $\mathrm{cm}$.) is one-tenth of that from the hottest part of the filament, then the potential difference between this point and the negative end is abont $0 \cdot 3$ volt for filaments of the diameter used. The fraction $1 / 10$ is of course chosen arbitrarily, but it probably gives the order of the quantity to be subtracted from the measured voitage to allow for the cooling due to the leads.

As regards the correction for the initial velocity of the electrons, it has been shown $\uparrow$ that the average kinetic energy of the emitted electrons is equal to $2 k \mathrm{~T}$, where $\mathrm{T}$ is the absolute temperature of the filament and $k$ is Boltzmann's constant. Now the average kinetic energy of the electrons within the filament is the same as that of a gas molecule at the same temperature, viz. $\frac{3}{2} k 1$. In the experiments here considered the value of $T$ for the central part of the filament was about $2500^{\circ} \mathrm{K}$, and at this temperature the value of $3 \mathrm{kT}$, expressed in equivalent volts, is about 0.33 . Hence $2 \mathrm{kT}$ is equivalent to about 0.44 volt. At the point on the filament,

* Stead, Journal of Inst. Elect. Engineers, vol, lviii. Jan. 1920, p. 107.

$\dagger 0$. W. Richardson, 'Emission of Electricity from Hot Bodies, pp. I40, 141. 


\section{Messrs. Stead and Gossling on Relative Ionization}

considered in the previous paragraph, where the electron emission is one-tenth of that corresponding to the central part of the filament, the temperature would be considerably lower than $2500^{\circ} \mathrm{K}$, and the correction would be reduced from 0.44 volt to about 0.40 volt. This correction must be added to the measured voltage, and it is seen to be almost compensated for by the amount $(0.3$ volt) to be subtracted on account of the non-uniformity of potential along the filament. It is to be observed that the velocities of the electrons emitted from a hot filament are distributed according to Maxwell's law, and the correction of 0.4 volt applies only to those electrons which are emitted with the average velocity corresponding to the given filament temperature. According to Maxwell's law of distribution an appreciable number of electrons, some 10 per cent. in fact, must come off with a velocity corresponding to at least one volt. It is clear that both this effect and also the want of uniformity of the potential of the filament must tend to make the position of the critical point in the current-voltage curves less sharply detined, and diminish the accuracy with which the ionization potential can be determined. Goucher * has overcome one of these difficulties by using as a source of electrons a platinum thimble heated to the required temperature by the radiation from a spiral of tungsten wire inside it, the tungsten wire being itself heated by an electric current. Such a source would not be satisfactory for the experiments here described because only a comparatively low temperature of the platinum is possible, and hence the electron emission is very small compared with that here employed.

The whole question of the contact difference of potential between a hot filament and a cold anode in a moderate or high vacuum appears to be obscure at the present time, and much work remains to be done before the correction to be applied to ionization potentials on this account can be computed with any degree of confidence. It seems quite likely, however, that a correction of the order of 0.5 volt may be necessary $\dagger$.

(5) Discussion of Results.-All these experiments on ionization potentials were carried out in May, June, and July, 1917, with the exception of those on helium, which were not undertaken until September 1917. Some of the

* Physical Review, viii. p. 561 (1916).

+ Richardson, 'Euisaion of Electricity from Hot Bodies'; Stoekle, Phys. Rev., viii. p. 534 (1916). 
results obtained were not in agreement with the values generally accepted at that time. Thus the ionization potentials of hydrogen and nitrogen, as measured by the Franck and Hertz method, came out at about 11 volts and 8 volts respectively, and for mercury vapour opinion was divided between 4.9 volts and about $10 \cdot 5$ volts. By a modification of the Franck and Hertz method, designed to eliminate photo-electric effects, Davis and Goucher ${ }^{*}$ have established the fact that no appreciable ionization occurs in mercury vapour under 10 volts. In the same paper Davis and Goucher came to the conclusion that ionization occurs in hydrogen at about 11 volts, but that a second type of ionization sets in at about 15.8 volts. Shortly after the appearance of Davis and Goucher's paper Bishopt published an account of some experiments on hydrogen in which he confirmed Davis and Goucher's result that a second (and stronger) ionization appears in hydrogen at between 15 and 16 volts. The higher value agrees with the value of the ionization potential of hydrogen as determined by the method described in the present paper. No trace of ionization at 11 volts, however, appears in a valve containing. hydrogen.

The only measurements of the ionization potential of nitrogen which are in agreement with the value here given $(17 \cdot 2$ volts $)$ are those made by Davis and Gouchert, and more recestly by H. D. Smyth $\S$. These observers have come to the conclusion that the true ionization potential of nitrogen is between 17 and 18 volts, and not about 8 volts, as had been previously supposed.

The value 12.5 volts for argon is in agreement with the result given by McLennan in the Physical Review for July 1917, but very recently Horton and Davies l' have come to the conclusion that ionization does not occur in argon until about 15 volts, and Rentschler $I$ gives the value 17 volt:. It is very difficult to reconcile this with the behaviour of argon in valves, in which it is hardly possible to avoid the conclusion that strong ionization occurs at about 12.5 volts.

The result (20) 8 volts) obtained for the ionization potential

* Physical Review, August 1917, p. 101.

+ Phys. Rev., September 1917, p.244.

$\$$ Phys. Rev., January 1919 .

$\$$ Phys. Rev. xiv. p. 409 (1919).

ii Proc. Roy. Soc. vol. xcvii. A. p. 1 (1920).

I Phys. Rev. xiv. p. 503 (1919). 


\section{Messrs. Stead and Gossling on Relative Ionization}

of helium is in agreement with that of most other observers*, except Horton and Daviest, who have recently found a higher value, as in the case of argon. Richardson and Bazzoni $\ddagger$ deduced a value of 29 volts from a consideration of the ultra-violet suectrum of helium.

As regards carbon monoxide, the value, 15 volts, here given at present lacks confirmation by other observers. Hughes and Dixon $\S$, employing a method of the Franck and Hertz type, obtained the value $7 \cdot 2$ volts. It seems probable that, as in the case of nitrogen, this low value represents a photo-electric effect, rather than the true ionization potential. At any rate there is no measurable ionization, in a valve containing carbon monoxide, below about 15 volts.

It may be of interest to note that the increase in slope of the current-voltage curve which occurs at the critical point is not accompanied by any visible radiation. The conditions for observing a faint glow between the electrodes are, however, not good, on account of the glare from the filament. All workers with soft valves are familiar with the fact that when the anode voltage exceeds a certain value a general glow suddenly fills the bulb, but the appearance of this general glow must on no account be confused with the critical point which has been taken by the writers to denote the formation of positive ions by collision. The general glow sets in at a much higher voltage than the critical pointusually not less than twice the voltage corresponding to the critical point. Moreover, whilst the position of the critical point is independent of the form of the electrodes and of the gas pressure, and nearly independent of the filament temperature, the potential at which the general glow appears depends very much on all these factors. Thus, in an audion type valve, with plane anodes and grids, the general glow occurs much more readily than in a eylindrical type valve, and the apprearance of the general glow depends so much on the gas pressure that a rough estimate of the softness of a valve may be made by observing the voltage at which the glow appears. Again, if a current-voltage curve is plotted up to a potential at which the general glow appears, there is at this point a complete discontinuity, the current suddonly jumping to many times its previous

* McLennan, Phys. Rev., July 1917, p. \$4. Bazzoni, loc. cit., and others.

+ Proc. Roy. Soc. vol. xerii. A, p. 408.

f Phil. Mag. xxxiv.p. 285 (1917).

$\$$ Phys. Rev., Norember 1917 . 
Potentials of Gases observed in Thermionic Valves. 425

value without, apparently, passing through any of the intermediate values. If the potential is now gradnally lowered there is a marked hysteresis effect, and the general glow suddenly disappears at a potential considerably lower than that at which it appeared. The disappearance of the general glow is accompanied by a sudden and discontinuous fall in the current. This behaviour is in marked contrast to the behaviour at the critical point. At this latter point there may be a discontinuity in the slope of the curve, but there is certainly no sudden jump in current without passing through intermediate values. Also there is little or no genuine hysteresis, the only observable effect being that the portion of the curve dependent on the $3 / 2$ power of the voltage may be slightly displaced, but not the position of the critical point. This displacement is probably due to some change in the surface of the filament resulting from its bombardment with positive ions.

The point at which the general glow appears also shows up in ordinary three-electrode valve characteristics as a sidden discontinuity in the value of the current, the occurrence of which makes the adjustment of some "soft" valves troublesome.

(6) Summary.-A simple method is described of determining the differences of potential which are necessary to cause positive ions to be produced in soft thermionic valves. This method has been applied to six different gases, viz.: mercury vapour, argon, hydrogen, carbon monoxide, nitrogen, and heliums.

The work was carried out in the Cavendish Laboratory, and the writers wish to express their best thanks to Professor Sir J. J. Thomson.

\section{Note added September 3, 1920.}

Since the above was wrioten another valve method of determining ionization potentials has heen described by Hodgson and Palmer *. For mercury vapour, nitrogen, and helium these observers obtained results substantially in agreement with those given in the present paper, but for argon a higher value $(16 \cdot 6$ volts $)$ was obtained.

* Radio Review, vol. i. Auc. 19:0, p. 525.

Phil. Mag. S. 6. Vol. 40. No. 238. Oct. 1920. 